$63^{\text {ème }}$ Congrès de la SFCO, 03021 (2015)

DOI:10.1051/sfco/20156303021

(C) Owned by the authors, published by EDP Sciences, 2015

\title{
Mise en place d'une démarche qualité en odonto-stomatologie : intérêts et freins rencontrés. Exemple de l'unité fonctionnelle de Chirurgie Orale, SCTD, Hospices Civils de Lyon
}

\author{
Bodard A-G**, Desoutter A*, Grammatica M*, Romero G** \\ * Service de Consultations et Traitements Dentaires, Hospices Civils de Lyon, 6 place Deperet, 69007 Lyon \\ ** Centre Léon Bérard Cervicofacial, Unit Surgery Department, 28 rue Laennec, Lyon
}

La mise en place d'une démarche qualité entre dans la thématique d'évaluation des pratiques professionnelles (HAS, XXX). L'amélioration de la qualité des soins, de leur efficience et de leur sécurité permet l'optimisation des pratiques. Si cette démarche est couramment répandue en milieu hospitalier médical, elle est plus rare dans le domaine de l'odonto-stomatologie. L'unité fonctionnelle de Chirurgie Orale du Service de Consultations et Traitements Dentaires (Hospices Civils de Lyon) développe cette démarche. Nous en verrons les intérêts, les avancées et les difficultés rencontrées.

La démarche qualité doit être mise en place de manière préventive, par la bonne tenue du dossier du patient et l'évaluation régulière de celui-ci (cahier de l’ADF, 2006). Le questionnaire médical est un des premiers éléments liés à la sécurité des soins. En pré-opératoire, des documents d'information venant compléter l'information orale sont remis au patient, un consentement éclairé est signé. Une check list préopératoire est effectuée avant tout acte chirurgical, y compris pour les actes effectués sous anesthésie locale. Des procédures concernant les tâches à effectuer et les étapes de réalisation sont mises en place et consultables aisément par le personnel de la structure de soin.

A posteriori, les événements indésirables doivent être analysés. Des fiches de déclaration d'événement indésirable sont à disposition (fiches ENNOV, Hospices Civils de Lyon, sur le réseau informatique et fiches de signalement papier propres à l'Unité). Ces fiches sont recueillies régulièrement et une sélection d'événements par analyse de criticité (fréquence $\mathrm{X}$ gravité) est faite de manière à hiérarchiser les événements. Un groupe de travail, ou comité de retour d'expérience (CREX), pluri professionnel (administration, assistante dentaire, praticien) sera mis en place afin de sélectionner un ou deux événements à analyser. L'analyse pourra être conduite en CREX ou en Réunion de Morbi-Mortalité (RMM). La méthologie de l'analyse est similaire : un pilote de l'analyse de l'événement est désigné, il va déterminer le déroulement de l'événement (méthode ORION ou 5M), présenter son analyse au CREX ou en RMM qui va ensuite proposer des actions correctives, désigner un responsable de la mise en place de ces actions et assurer un suivi de ces actions. Ainsi, les barrières et verrous à la survenue d'un événement indésirable ou d'un précurseur à un événement sont renforcés. Le patient et le praticien y gagnent en sécurité, efficience, qualité des soins et cette démarche s'inscrit dans le Développement Professionnel Continu.

This is an Open Access article distributed under the terms of the Creative Commons Attribution License 4.0, which permits unrestricted use, distribution, and reproduction in any medium, provided the original work is properly cited. 
$63^{\text {ème }}$ Congrès de la SFCO

Nom et adresse du conférencier

Anne-Gaëlle BODARD

Centre Léon Bérard Cervicofacial Unit Surgery Department 28 rue Laennec

Lyon (France)

anne-gaelle.bodard@lyon.unicancer.fr 